\title{
COMPARAÇÃO DO USO DE APLICAÇÃO AÉREA E TERRESTRE NA QUALIDADE DE DEPOSIÇÃO DE PRODUTO NA CULTURA DA SOJA
}

\author{
Thiago Martins Machado ${ }^{1}$, Étore Francisco Reynaldo ${ }^{2}$ \\ ${ }^{1}$ Professor Adjunto do Instituto de Ciências Agrárias e Ambientais da Universidade \\ Federal de Mato Grosso - Sinop - MT - Brasil \\ E-mail: tmmachado@ufmt.br \\ ${ }^{2}$ Gerente de manutenção da Syngenta - Uberlândia - MG - Brasil

\section{Recebido em: 06/04/2019 - Aprovado em: 10/06/2019 - Publicado em: 30/06/2019 DOI: 10.18677/EnciBio_2019A37}

\begin{abstract}
RESUMO
O objetivo do presente trabalho foi a avaliação da qualidade de aplicação de produto fitossanitário nas plataformas aérea e terrestre na cultura da soja. Na plataforma de aplicação terrestre foi utilizado o pulverizador autopropelido marca John Deere, modelo JD 4730 o volume de calda utilizado foi de $100 \mathrm{~L} \mathrm{ha}^{-1}$, velocidade de deslocamento de $14 \mathrm{~km} \mathrm{~h}^{-1}$ e pressão de trabalho de 4.8 bar. A aplicação aérea foi realizada com o avião agrícola marca Embraer, modelo Ipanema equipado com jato atomizador modelo Micronair AU-5000, com volume de calda foi de $20 \mathrm{~L} \mathrm{ha}^{-1}$. Os produtos utilizados para associação no controle de ferrugem asiática da soja foram: Aureo $0,3 \mathrm{~L} \mathrm{ha}^{-1}$, Fox $0,35 \mathrm{~L} \mathrm{ha}^{-1}$, e TA35 $0,033 \mathrm{~L} \mathrm{ha}^{-1}$, a aplicação foi realizada no estádio fenológico reprodutivo $R$ 5.5. O ensaio foi realizado em delineamento experimental inteiramente casualizado com nove repetições. Foram avaliados a deposição do produto nos terços superior, médio e inferior da cultura, com a utilização de papel hidro sensível, marca Teejet e diâmetro de 26x76 mm. Foi possível identificar diferença estatística entre a aplicação terrestre e aplicação aérea em relação ao DMV no terço superior da planta. Nas demais posições não houve diferença. A aplicação aérea apresentou menor amplitude relativa no terço inferior quando comparado à aplicação terrestre, o que demonstra maior uniformidade de tamanho de gota da aplicação aérea. O número de gotas e área de cobertura foram maiores no tratamento que utilizou a aplicação terrestre.
\end{abstract}

PALAVRAS-CHAVE: Avião, tecnologia de aplicação, volume de aplicação

\section{COMPARISON OF THE USE OF AIR AND TERRESTRIAL APPLICATION IN THE QUALITY OF PRODUCT DEPOSITION IN SOYBEAN CULTURE}

\begin{abstract}
The objective of this study was to evaluate the phytosanitary product application quality on air and land platforms in soybeans. In the terrestrial application platform self-propelled sprayer was used John Deere brand, model 4730 JD the volume of slurry used was $100 \mathrm{~L} \mathrm{~h}^{-1}$, velocity of $14 \mathrm{~km} \mathrm{~h}^{-1}$ and 4.8 bar work pressure. The aerial application was carried out with agricultural aircraft Embraer brand,
\end{abstract}


Ipanema model equipped with jet atomizer Micronair AU 5000 model with spray volume was $20 \mathrm{~L} \mathrm{ha}^{-1}$. Products used for membership in the Asian rust control soybean were Aureo $0.3 \mathrm{~L} \mathrm{ha}^{-1}, 0.35$ Fox $L$ ha $^{-1}$, and TA35 $0.033 \mathrm{~L} \mathrm{ha}^{-1}$, it was used in the reproductive growth stage $R 5.5$. The test was conducted in a completely randomized design with 9 repetitions. They evaluated the deposition of the product in the upper, middle and lower culture with the use of water sensitive paper, Teejet brand and $26 \times 76 \mathrm{~mm}$ diameter. It was possible to identify statistically significant differences between terrestrial application and the air application relative DMV in the upper third of the plant. In the other positions there was no difference. Aerial application had lower relative amplitude in the lower third compared to terrestrial application, which shows greater uniformity of aerial application of droplet size. The number of drops and coverage area were higher in treatment used terrestrial application.

KEYWORDS: Plane, application technology, application volume

\section{INTRODUÇÃO}

A utilização de agrotóxicos para atingir bons níveis de produtividade na agricultura convencional tem sido indispensável, uma vez que, a incidência de pragas, doenças e plantas daninhas é expressiva (RUAS et al., 2014). A cultura da soja pode ser atacada por mais de 40 doenças, sendo que a ferrugem asiática da soja (Phakopsora pachyrhizi Sydow e Sydow) é a mais importante, podendo causar grandes perdas, principalmente devido à desfolha precoce (FIALLOS, 2011).

O controle da ferrugem asiática não é simples devido, principalmente, ao rápido espalhamento, agressividade e também devido às próprias características da cultura, que apresenta grande massa foliar nos estádios reprodutivos dificultando a penetração das gotas durante os tratamentos fitossanitários (MACHADO; REYNALDO, 2016). Dentre as alternativas utilizadas para favorecer o controle da doença, trabalhar com gotas finas tanto nas aplicações aéreas quanto terrestres, tem se mostrado viável, já que as gotas finas apresentam maior facilidade de penetração no dossel da cultura (CARVALHO et al., 2013). O controle efetivo de defensivos na agricultura, está relacionado tanto na eficácia desses produtos, quanto na tecnologia de aplicação utilizada no controle sobre pragas, doenças e plantas daninhas (REYNALDO; MACHADO, 2017).

As aplicações com fungicidas e a cobertura proporcionada sobre o dossel da planta de soja, são em geral, desuniformes e principalmente no que se refere à parte inferior da planta, apresentando então controle insuficiente mesmo quando são usados produtos sistêmicos. A tecnologia de aplicação de produtos fitossanitários é uma ferramenta que pode ser usada para potencializar a produtividade. Nesse contexto, tem-se a aplicação aérea que é uma realidade em boa parte das regiões produtoras de grãos no Brasil, sendo uma alternativa para grandes áreas e intervalos de aplicações curtos (CUNHA et al., 2014).

A aviação agrícola brasileira está em franca expansão, sendo a segunda maior frota do mundo. Entrou no ano de 2019 com 2.194 aeronaves, segundo (SINDAG, 2019), o resultado significa um crescimento de 79 aeronaves em 2018, registrando alta de $3,74 \%$ durante o ano, mais do que o dobro dos 1,54\% (32 aviões) que a frota havia crescido em 2017.

A utilização de aeronaves agrícolas na substituição de autopropelidos na aplicação sobre as principais culturas nacionais (soja e milho) tem aumentado pelo fato, de se ter maior rendimento na aplicação, principalmente em grande escala de áreas. Regionalmente, a aplicação aérea de 
fungicidas na cultura do milho é uma realidade, principalmente pela característica morfológica das plantas, no impedimento físico na entrada de equipamentos terrestres na lavoura (CUNHA et al., 2014).

Perdas em produtividade devido aos danos causados pelo tráfego de pulverizadores terrestres são relatadas por Carvalho et al., (2013), que afirmam que os danos por amassamento de plantas e a compactação do solo durante o ciclo das culturas estão em torno de 5 a $10 \%$; O principal fator que delimita a utilização do manejo aéreo é a qualidade de aplicação, relacionado com o volume de calda e dose de ingrediente ativo, além do modo de ação, que deve atingir o alvo especifico nas plantas.

Uma das maiores dificuldades da área de tecnologia de aplicação é de alcançar as partes do baixeiro das plantas, onde se concentra a maioria das doenças e onde se deve aplicar com prioridade, para o combate mais eficiente contra as pragas (CUNHA et al., 2014; MACHADO, 2014).

As condições ambientais como a temperatura, umidade e vento são responsáveis pelo sucesso na aplicação tanto na terrestre como na aérea. A temperatura ideal não superior a $30^{\circ} \mathrm{C}$, umidade maior que $50 \%$ e ventos entre 3 a $10 \mathrm{~km} \mathrm{~h}^{-1}$ devem ser respeitados (ANDEF, 2014). Estas condições ambientais ideais são a garantia de sucesso no controle fitossanitário.

Com a elevação dos custos de produção e a maior competitividade do processo de globalização das economias a engenharia de aplicação tem como principal objetivo aumentar a eficácia da aplicação de produtos químicos para o controle de pragas, com a aplicação dos conhecimentos científicos para a correta colocação de um produto biologicamente ativo no alvo, em quantidade necessária, de forma econômica e com mínimo de contaminação de outras áreas (VAN ZYL et al., 2013). O objetivo deste trabalho foi a avaliação da qualidade de aplicação de produto fitossanitário nas plataformas aérea e terrestre na cultura da soja.

\section{MATERIAL E MÉTODOS}

O ensaio foi conduzido na Fazenda Cupim, propriedade do Grupo Reinhofer, no munícipio de Guarapuava - PR, localizada na seguinte coordenada geográfica: latitude $-25^{\circ} 32^{\prime} 16.23^{\prime \prime}$ e longitude $-51^{\circ} 34^{\prime} 54.45^{\prime \prime}$. Altitude de 1057 metros em relação ao nível médio dos mares.

A cultivar de soja semeada no local do ensaio foi a cultivar Ativa RR, semeada em 12/11/2014. A densidade populacional foi de 400 mil plantas por hectare. A adubação de base de $400 \mathrm{~kg} \mathrm{ha}^{-1}$ na formulação 08-30-20 e $200 \mathrm{~kg} \mathrm{ha}^{-1}$ de $\mathrm{KCl}$ em cobertura.

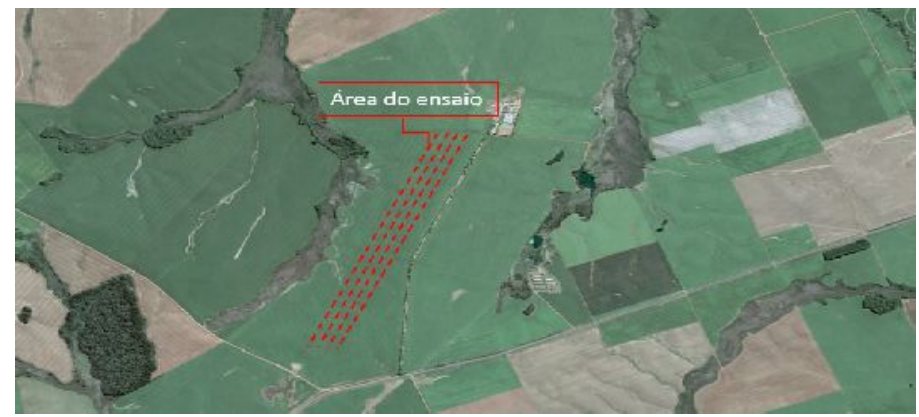

FIGURA 1 - Local do ensaio de avaliação de deposição de gotas através de plataforma terrestre e aérea. 
Na plataforma de aplicação terrestre foi utilizado o pulverizador autopropelido marca John Deere, modelo JD 4730 com 30 metros de barra com porta bicos espaçados a $0,5 \mathrm{~m}$ e conjunto de pontas do tipo leque simples de jato plano, modelo GRD 120 03. O volume de calda utilizada foi de $100 \mathrm{~L} \mathrm{ha}^{-1}$, velocidade de deslocamento de $14 \mathrm{~km} \mathrm{~h}^{-1}$ e pressão de trabalho de 4,8 bar. A aplicação aérea foi realizada com o avião agrícola marca Embraer, modelo Ipanema equipado com jato atomizador modelo Micronair AU-5000. O volume de calda foi de $20 \mathrm{~L} \mathrm{ha}^{-1}$.

Os produtos utilizados para associação no controle de ferrugem asiática da soja foram: Áureo $0,3 \mathrm{~L} \mathrm{ha}^{-1}$, Fox $0,35 \mathrm{~L} \mathrm{ha}^{-1}$, e TA35 0,033 $\mathrm{L} \mathrm{ha}^{-1}$, a aplicação foi realizada no estádio fenológico reprodutivo $R$ 5.5. No Quadro 1 são apresentados os tratamentos utilizados no ensaio de comparação do uso de aplicação aérea e terrestre nos atributos de qualidade de deposição de produto no alvo.

QUADRO 1 - Tratamentos.

\begin{tabular}{cl}
\hline$N^{\circ}$ Tratamento & \multicolumn{1}{c}{ Descrição } \\
\hline 1 & Aplicação terrestre (Autopropelido JD4730) \\
2 & Aplicação aérea (Ipanema) \\
\hline
\end{tabular}

O ensaio foi realizado em delineamento experimental inteiramente casualizado com nove repetições. Foram avaliadas as deposições de calda nos terços das plantas de soja, superior, médio e inferior, com a utilização de papel hidro sensível, marca Teejet e diâmetro de 26x76 milímetros. Os terços foram divididos conforme a estatura da planta de soja: terço superior (Ts), terço médio $(\mathrm{Tm})$ e terço inferior (Ti), dispostos a 0,8; 0,5 e 0,2 metros do solo respectivamente.

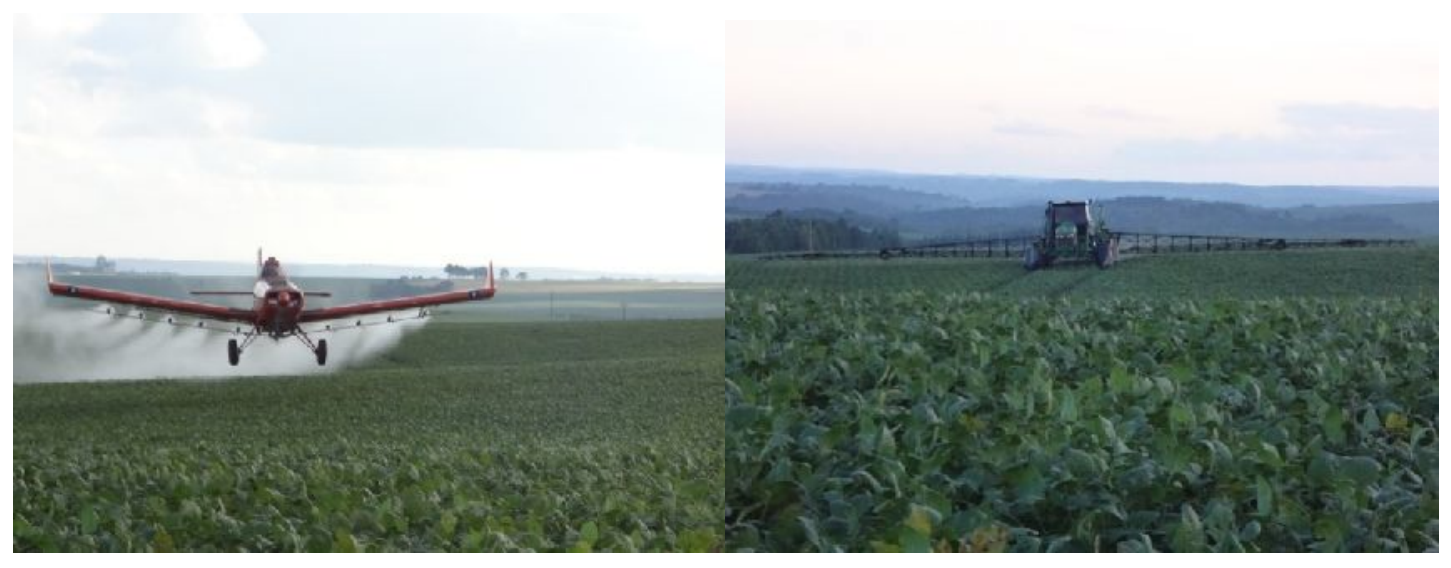

FIGURA 2 - Aplicação Aérea e Terrestre

O horário da aplicação aérea iniciou as 17:20 h e teve término as 17:30 h. A aplicação terrestre iniciou no horário das $18: 50 \mathrm{~h}$ e teve término as 19:10 h. Após a pulverização os papéis foram retirados e armazenados em local apropriado e posteriormente analisados no software Adobe Photoshop CS2 e e-Sprinkle.

O conjunto de parâmetros analisados foram: amplitude relativa, o diâmetro mediano numérico (DMN) e o diâmetro mediano volumétrico (DMV). As análises estatísticas foram realizadas utilizando o programa SAS 9.3. Primeiramente foi aplicado teste de homogeneidade de variância. Após, os resultados obtidos foram submetidos à estatística pelo teste $F$ e as médias foram comparadas pelo teste Tukey ao nível de $5 \%$ de significância. 


\section{RESULTADOS E DISCUSSÃO}

$\mathrm{Na}$ Tabela 1 têm-se os dados climáticos no momento da realização do ensaio. A umidade relativa do ar registrada durante a realização das aplicações foram de 70,0 e $71,4 \%$ para o primeiro e segundo tratamento respectivamente.

TABELA 1 - Dados climáticos dos tratamentos de aplicação do ensaio.

\begin{tabular}{cccc}
\hline$N^{\circ}$ Tratamento & Umidade $(\%)$ & Temperatura $\left({ }^{\circ} \mathrm{C}\right)$ & Velocidade do Vento $\left(\mathrm{km} \mathrm{h}^{-1}\right)$ \\
\hline 1 & 70,0 & 24 & 3,5 \\
2 & 71,4 & 26 & 2,9 \\
\hline
\end{tabular}

A temperatura variou de 24 a $26^{\circ} \mathrm{C}$, na média a velocidade do vento variou de 3 a $5 \mathrm{~km} \mathrm{~h}^{-1}$. A condição climática foi considerada dentro dos padrões (recomendação) apara a realização da aplicação de produtos fitossanitários. No Quadro 2 estão apresentados os papéis hidro sensíveis coletados em um dos pontos de controle para as aplicações realizadas com o auto propelido e com o avião.

QUADRO 2 - Papel hidro sensível coletado no ponto de controle 4 nos 3 terços da planta.

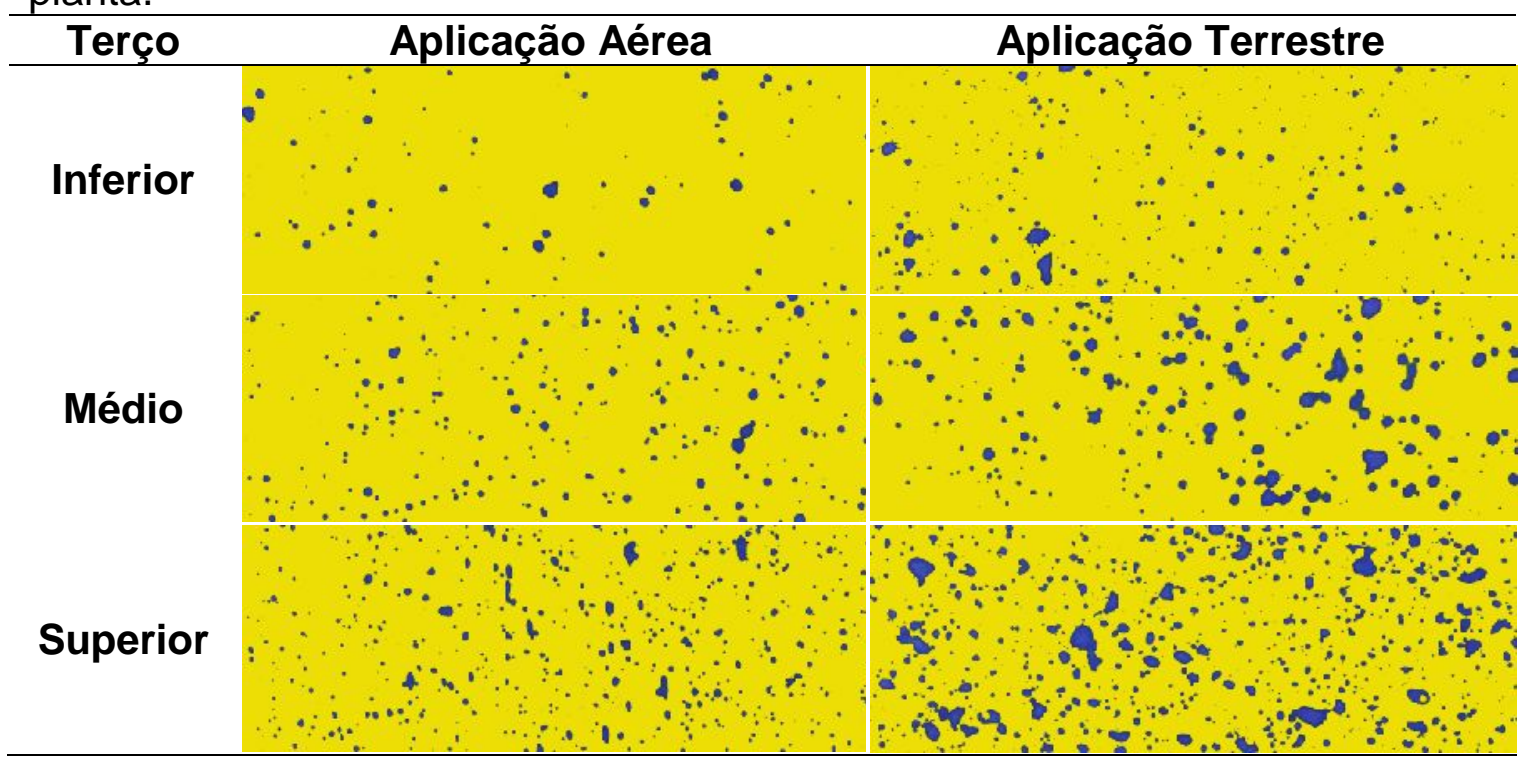

De maneira geral, a deposição dos pesticidas aplicados na pulverização é menor nas partes mais baixas e internas do dossel das culturas (REYNALDO; MACHADO, 2017). Segundo Cunha et al. (2014) tanto a aplicação aérea quanto terrestre proporcionaram a deposição mínima recomendada para aplicação de fungicida. Na Figura 3 têm-se os dados do diâmetro mediano volumétrico (DMV) proveniente da análise de papel hidro sensível, dispostos nos terços superior, médio e inferior, em função do modo de aplicação, aérea e terrestre. 


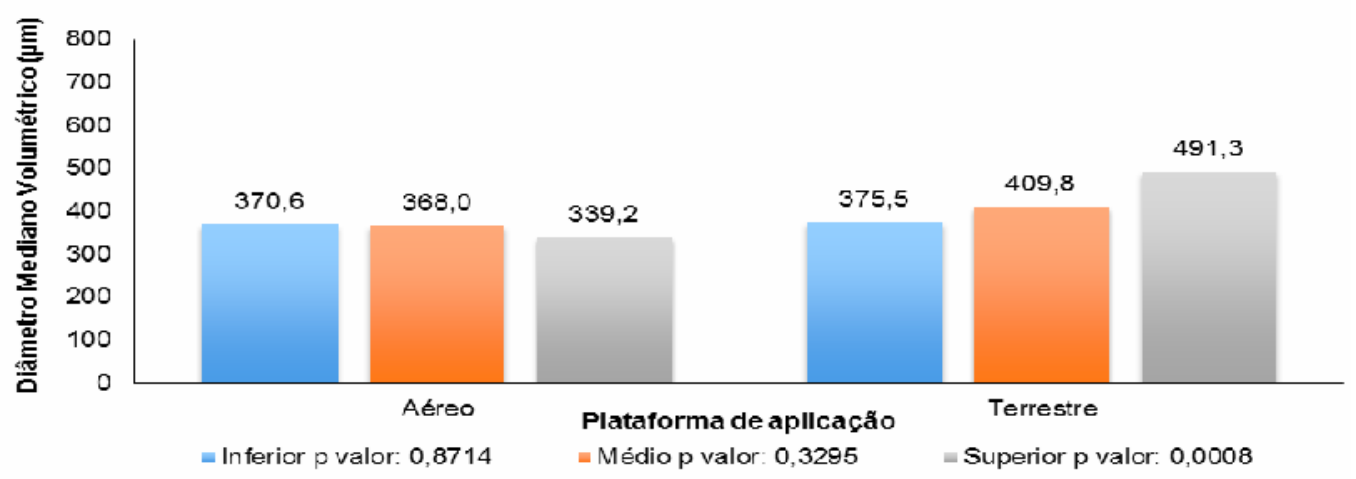

FIGURA 3 - Diâmetro mediano volumétrico (DMV) por posição (terço).

Foi possível estabelecer diferenças estatísticas entre os tratamentos T1 (aplicação terrestre) e T2 (aplicação aérea) quando analisado o DMV no terço superior (Ts) da planta de soja. No Ts apresentou DMV de 339,21 e 491,34 $\mu \mathrm{m}$ dos tratamentos T2 e T1 respectivamente. O maior DMV da gota no Ts é devido à forma de fragmentação da gota, consequência do modelo de ponta adotado. Analisando ainda o comportamento do DMV, verifica-se que a aplicação aérea apresenta uma maior uniformidade do tamanho da gota, sendo que na terrestre as gotas apresentam DMV maior no Ts, apresentando tendência de diminuir no Tm e Ti. Vale frisar que um DMV muito alto, também não é vantajoso, pelo fato de causar escorrimento do produto aplicado sobre $o$ alvo.

Na Figura 4 é apresentado $D_{v 0,1}$ do comparativo entre os tratamentos, $D_{v 0,1}$ é o diâmetro de gota tal que $10 \%$ do volume do líquido pulverizado é constituído de gotas de tamanho menor que esse valor. Não houve diferença estatística em relação as posições Ti e Tm. Porém no Ts foi observada diferença estatística ao nível de 5\% de probabilidade.

$\mathrm{D}_{\mathrm{v} 0,1}$ com diâmetro abaixo $100 \mu \mathrm{m}$ pode indicar a presença de deriva na área, pois, as gotas com o diâmetro menor que 100 tem massa pequena de fácil arraste pelas correntes convexas, consequentemente, o tempo de vida da gota é muito pequena e o produto acaba por não atingir o alvo.

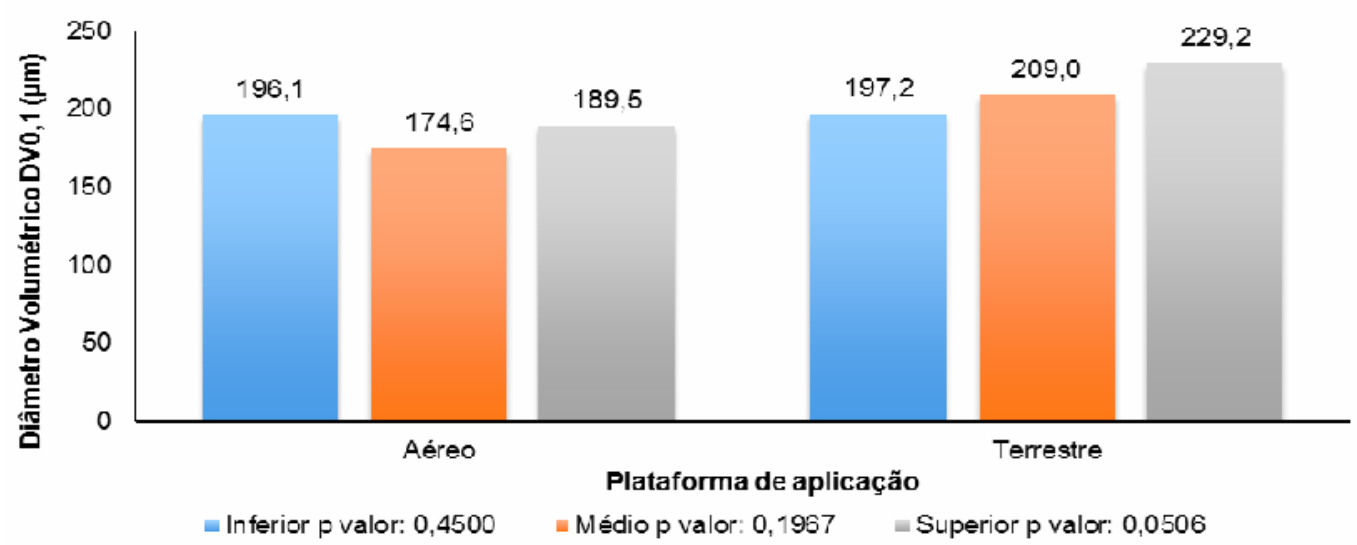

FIGURA 4 - Diâmetro $D_{v 0,1}(\mu \mathrm{m})$ - 10\% do volume pulverizado em gotas menores que o DV.

$\mathrm{Na}$ Figura 5 tem-se o diâmetro de gota tal que $90 \%$ do volume do líquido pulverizado é constituído de gotas de tamanho 
menor que esse valor $\left(D_{\mathrm{v}, 9}\right)$ apresentando diferença estatística entre tratamentos no Ts e Tm. Este resultado complementa a afirmação sobre o processo de fragmentação de gotas e o padrão gerado na aplicação aérea tendo melhor uniformidade de gota entre os três terços da planta, quando comparada ao terrestre.

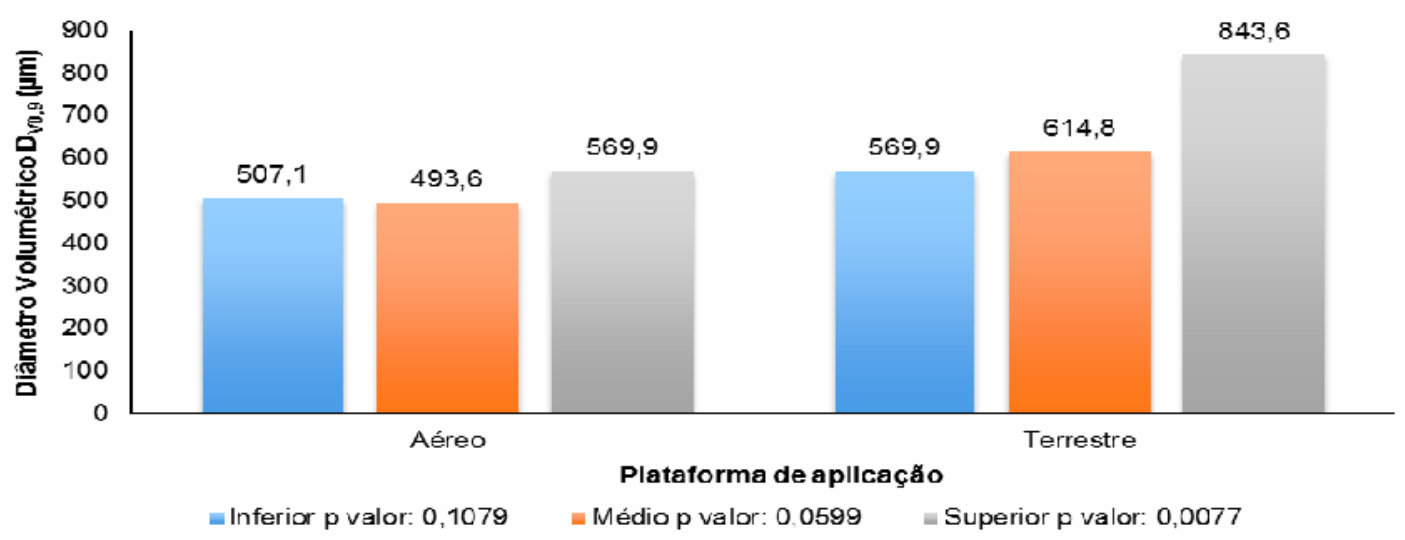

FIGURA 5 - Diâmetro $D_{\mathrm{v} 0,9}(\mu \mathrm{m})$ - 90\% do volume pulverizado em gotas menores que o DV.

Na Figura 6 são apresentados os dados de amplitude relativa no espectro de gotas aplicados pela pulverização terrestre e aérea. A amplitude relativa indica a homogeneidade do espectro de gotas. Espectro de gotas homogêneo tem valor de amplitude relativa tendendo a zero.

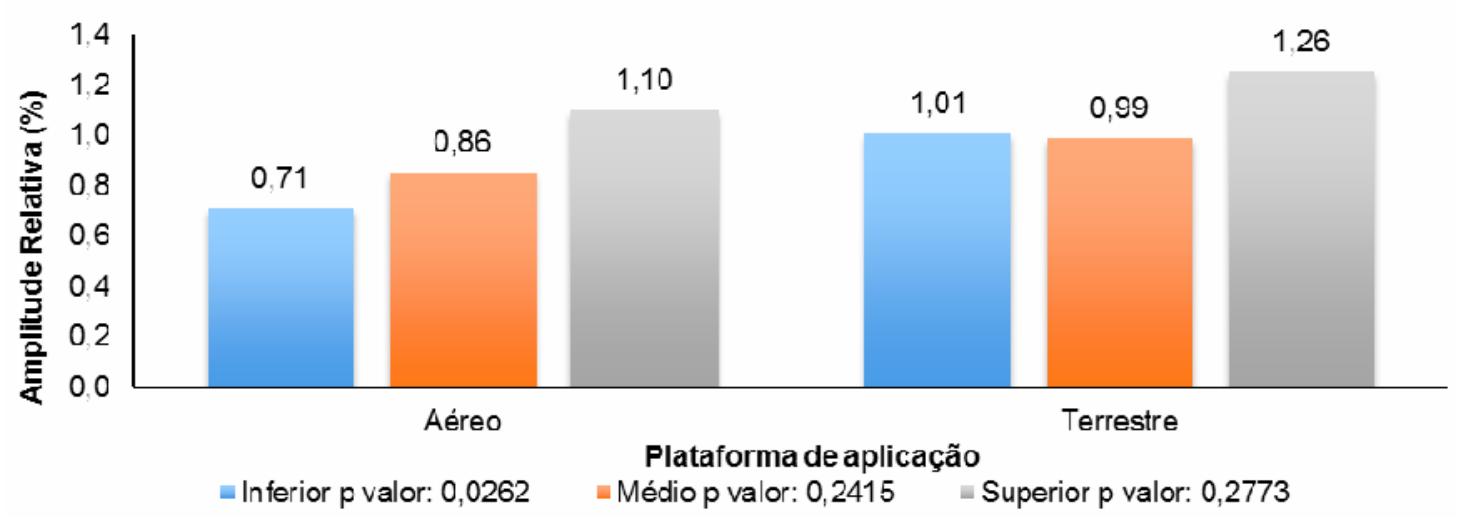

FIGURA 6 - Dados de amplitude relativa nas diferentes posições de deposição de produto.

Entre os modos de aplicação houve diferença significativa para a posição Ti, sendo a aplicação aérea com menor variação no terço inferior quando comparada a aplicação terrestre. Esta diferença está ligada a forma de fracionamento do liquido em gota, como já falado anteriormente, resultando em maior homogeneidade (padrão) das gotas, em outras palavras, gotas com o mesmo tamanho. Em avaliação da uniformidade de distribuição da calda, proporcionada pelas aplicações terrestres 
e aéreas cunha et al., (2014) concluíram que a uniformidade foi baixa, principalmente na parte inferior da cultura.

Segundo Da Costa (2009) as aplicações de fungicida por via aérea apresentam performance superior, quando comparadas com aquelas realizadas por via terrestre, sendo os danos por amassamento causados pelo pulverizador terrestre um fator relevante no momento de comparar as duas técnicas. Na Figura 7 é apresentado o coeficiente de variação para o DMV nas diferentes posições em função do modo de aplicação.

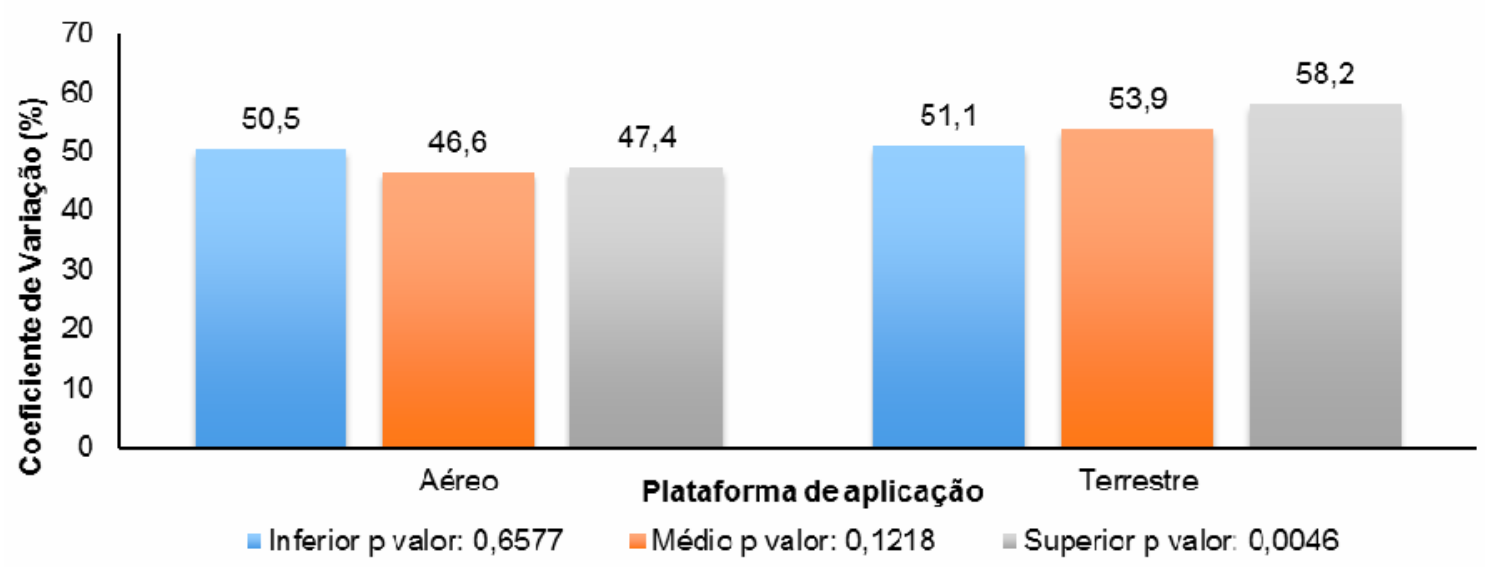

FIGURA 7 - Coeficiente de variação de DMV.

Foi observada diferença estatística somente para o coeficiente de variação do terço superior, quando a aplicação via tecnologia aérea obteve menor variação no tamanho de gota. Nas demais posições os tratamentos não se diferenciaram. Diferentemente do apresentado na Figura 7 o número de gotas foi estatisticamente diferente para as posições Ts e Tm de deposição do produto. Como pode ser visualizado no gráfico da Figura 8. A aplicação terrestre apresentou incremento em relação à aplicação aérea nessas duas posições de deposição de produto avaliadas.

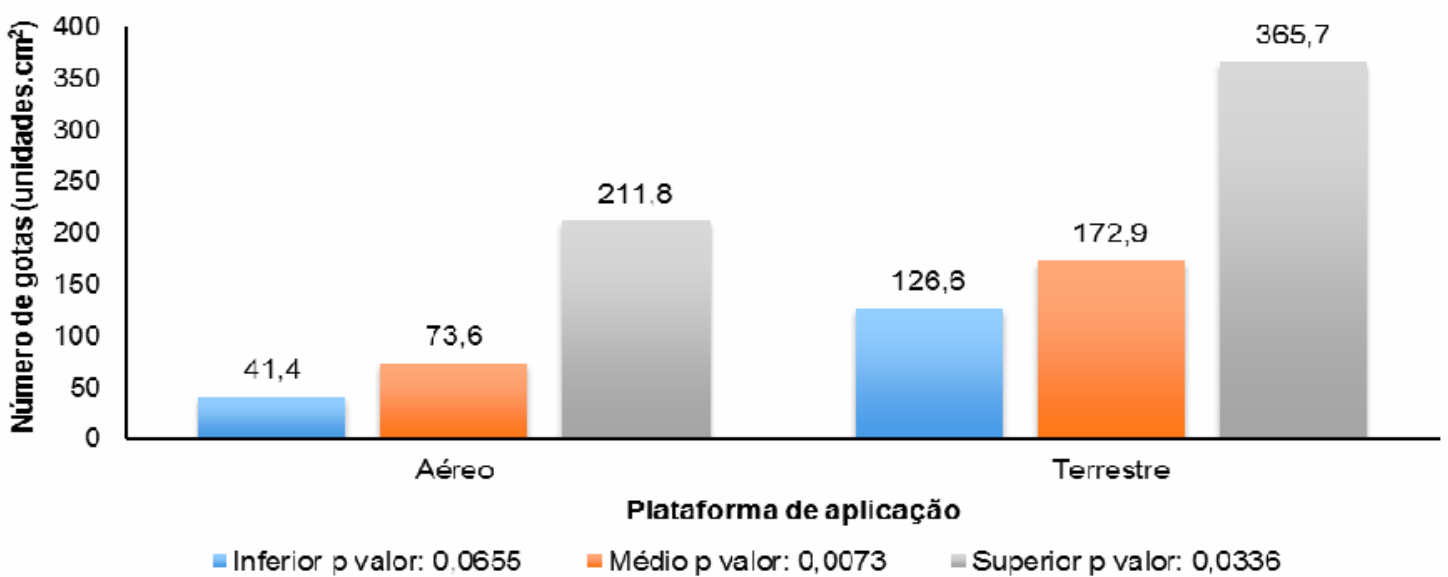

FIGURA 8 - Número de gotas nas diferentes posições em função do modo de aplicação. 
No gráfico da Figura 9 é apresentada a área de cobertura nas diferentes posições das plantas avaliadas em função do modo de aplicação. $O$ tratamento utilizando a aplicação terrestre apresentou os melhores resultados quando comparados ao modo de aplicação aéreo. Vale frisar que o volume de aplicação do modo terrestre foi de $100 \mathrm{~L} \mathrm{ha}^{-1}$ e da aérea foi de $20 \mathrm{~L} \mathrm{ha}{ }^{-1}$. Carvalho et al. (2013) avaliando a quantidade de calda depositada utilizando adjuvante e óleo mineral juntamente com fungicida, não observaram diferenças entre aplicação aérea e terrestre.

Ao longo das avaliações identificou-se que os modos de aplicação terrestre e aéreo se alternam dependendo do atributo avaliado, sendo as diferenças estatísticas quando observadas, apresentando valores muito próximos. Com isso pode-se dizer que ambas as tecnologias de aplicação podem ser utilizadas de forma a otimizar o rendimento agrícola. Contudo, o principal fator que pode prejudicar ambas as tecnologias de aplicação diz respeito às condições climáticas que podem interferir na eficiência e qualidade de aplicação (REYNALDO et al.,2015).

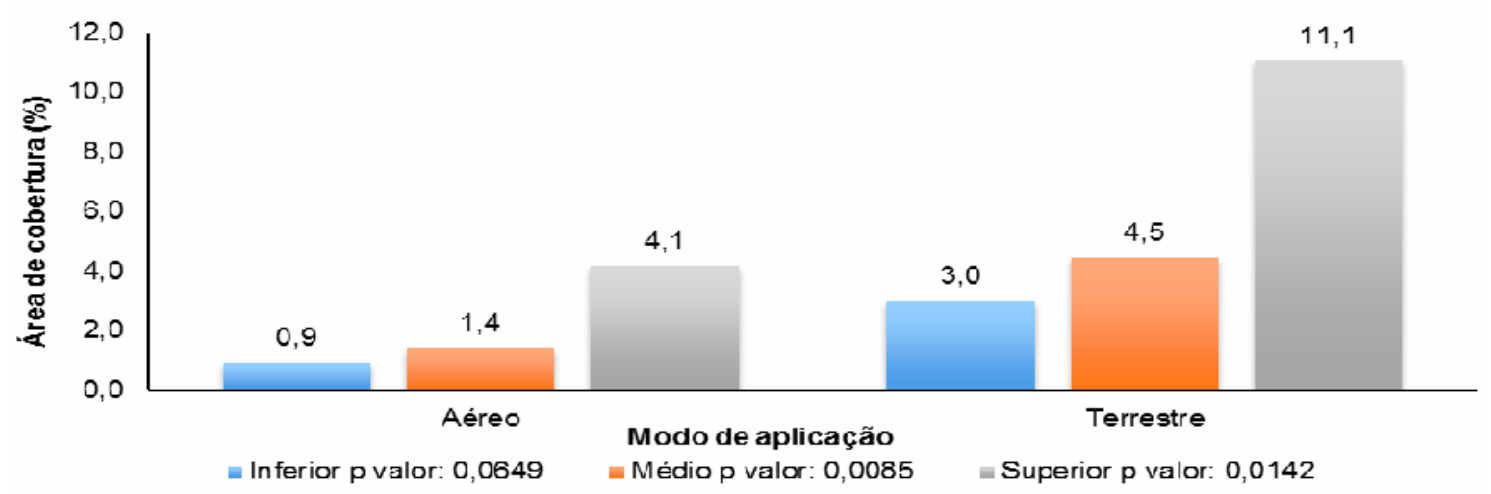

FIGURA 9 - Área de cobertura nas diferentes posições em função do modo de aplicação.

De maneira geral, a aplicação aérea apresentou resultados bastante semelhantes aos da aplicação terrestre no que diz respeito ao tamanho de gota. No entanto, a quantidade em volume de gotas no tratamento utilizando a aplicação terrestre apresentou os melhores resultados quando comparados ao modo de aplicação aéreo, o que se deve ao maior volume de calda. Teoricamente, em condições climáticas ideais, esse fator não deve alterar a eficiência da aplicação, sendo que a concentração do ingrediente ativo também é maior na aplicação aérea. Em condições climáticas inadequadas, esse menor volume de calda pode apresentar uma criticidade maior em relação à aplicação terrestre.

\section{CONCLUSÕES}

Foi possível identificar diferença entre a aplicação terrestre e aplicação aérea em relação ao DMV no terço superior da planta e nas demais posições não houve diferença.

A aplicação aérea apresentou menor amplitude relativa no terço inferior quando comparado à aplicação terrestre, o que demonstra maior uniformidade de tamanho de gota da aplicação aérea.

O número de gotas e área de cobertura foram maiores no tratamento que utilizou a aplicação terrestre. 
Novos ensaios são necessários para ratificação dos dados, visto que as diferenças identificadas foram muito próximas entre os diferentes tratamentos.

A aplicação aérea torna-se uma alternativa bastante interessante, principalmente em situações de necessidade de alto rendimento operacional, como nos casos em que se perde o ponto ideal de aplicação.

\section{REFERÊNCIAS}

ANDEF - Associação Nacional de Defesa Vegetal . Manual de tecnologia de aplicação de produtos fitossanitários. São Paulo: Línea Creativa, 2014. 76 p.

CARVALHO, F. KASSIS, ANTUNIASSI, U. R.; MOTA, A. A. BARBIERI.; CHECHETTO, R. G.; GANDOLFO, U. D. Adjuvantes na deposição de aplicações aéreas e terrestres. Energia na Agricultura. Botucatu, v. 28, n.4, p.215-221, 2013.

Disponível em: < file:///C:/Users/tmmac/Downloads/857-Texto\%20do\%20artigo4908-2-10-20140123.pdf > Acesso em: 15 janeiro. 2019.

CUNHA, J. P.; JULIATTI, F. C.; REIS, E. Tecnologia de aplicação no controle da ferrugem asiática da soja: resultados de oito anos de estudos em Minas Gerais e Goiás. Bioscience Journal, Uberlândia, v.30, n. 4, p.950-957, Junho/ Aug. 2014. Disponível em: <http://www.seer.ufu.br/index.php/biosciencejournal/article/viewFile/20907/14739 > . Acesso em: 09 janeiro. 2019.

DA COSTA, D. I. Eficiência e qualidade das aplicações de fungicidas, por vias terrestre e aérea, no controle de doenças foliares e no rendimento de grãos de soja e milho. 126 f. Tese (Doutorado em Agronomia) - Universidade de Passo Fundo: Passo Fundo. 2009.

FIALLOS, F. R. G. A ferrugem asiática da soja causada por Phakopsora pachyrhizi Sydow e Sydow. Ciencia y Tecnología, Garcés, v. 4, n. 2, p. 45-60, 2011.

MACHADO, T. M.; REYNALDO, E. F. Avaliação de pontas e sistema de barras auxiliares para controle da Sclerotinia sclerotiorum na cultura da soja. Enciclopédia biosfera, Centro Científico Conhecer - Goiânia, v.13 n.23, p. 148-154, 2016. Disponível em: <http://www.conhecer.org.br/enciclop/2016a/agrar.htm> DOI: 10.18677/Enciclopedia_Biosfera_2016_013

MACHADO, T. M. Inspeção periódica de pulverizadores de barras na região de guarapuava - pr. Enciclopédia biosfera, Centro Científico Conhecer - Goiânia, v.10, n.19; p. 1225-1233, 2014. Disponível em: https://docplayer.com.br/39095323Inspecao-periodica-de-pulverizadores-de-barras-na-regiao-de-guarapuava-pr-thiagomartins-machado.html

REYNALDO, E. F.; MACHADO, T. M. Performance of spray nozzles to control fusarium head blight and mycotoxin in the barley crop. Revista Brasileira. Engenharia Agrícola e Ambiental, Campina Grande, v.21, n.3, p.209-213, 2017. Disponível em: < http://www.scielo.br/scielo.php?script=sci_arttext\&pid=S141543662017000300209> Acesso: 06 abril 2019, DOI: dx.doi.org/10.1590/18071929/agriambi.v21n3p209-213 
REYNALDO, E. F.; MACHADO, T. M , TAUBINGER, L.; QUADROS. D.Avaliação de horários alternativos para aplicação de fungicida Para o controle da ferrugem asiatica da soja. Enciclopédia biosfera, Centro Científico Conhecer - Goiânia, v.11 n.22; 427-433, $2015 \quad$ DOI: http://dx.doi.org/10.18677/Enciclopedia_Biosfera_2015_096

RUAS, R. A. A.; SICHOKI, D; GOD, P. I. V. G.; CARVALHO FILHO, A.; RONCHI, C. $P$. Condições operacionais das aplicações de agrotóxicos empregando-se pulverizadores hidráulicos. Global Science Technology, Rio Verde, v. 07, n. 01, p.115-128. 2014.

SINDAG. Disponível em: < https://www.agrolink.com.br/noticias/brasil-tem-segundamaior-frota-de-avioes-agricolas-do-mundo_416226.html >. Acesso: 06 abril 2019.

VAN ZIL, J. G.; FOURIE, P. H.; SCHUTTE, G. C. Spray deposition assessment andbenchmarks for control of Alternaria brown spot on mandarinleaves with copper oxychloride. Crop Protec. v. 46, n. 4.p. 80-87, 2013. 